\title{
Identification of urinary hsa_circ_0137439 as a potential biomarker and tumor regulator of bladder cancer
}

\author{
Z. SONG 1 , Q. ZHANG 2 , J. ZHU², G. YIN ${ }^{2}$, L. LIN², C. LIANG ${ }^{1, \star}$ \\ ${ }^{1}$ Department of Urology, First Affiliated Hospital of Anhui Medical University, Hefei, China; ${ }^{2}$ Department of Urology, Taizhou First People's \\ Hospital, Taizhou, China \\ ${ }^{*}$ Correspondence: Liang_chaozhao@163.com
}

Received December 14, 2018 / Accepted August 14, 2019

\begin{abstract}
Cell-free circular RNAs (circRNAs) stably and abundantly exist in body fluids. In this study we aimed to investigate the potential of urinary cell-free circRNAs as a novel class of non-invasive disease biomarkers for diagnosis of bladder cancer. Differentially expressed circRNAs from 10 normal and 10 bladder cancer urine samples were firstly detected by microarray. Hsa_circ_0137439 was then screened and validated in 30 normal and 116 bladder cancer samples. A receiver operating characteristic (ROC) curve was used to evaluate the diagnostic value of hsa_circ_0137439. The Kaplan-Meier method was used to evaluate the significance of hsa_circ_0137439 in the prognosis of bladder cancer. We found that hsa_circ_0137439 was significantly upregulated in bladder cancer samples. Moreover, increased expression of hsa_circ_0137439 was correlated with higher tumor stage, higher tumor grade, higher lymph node status, and history of muscle-invasive bladder cancer (MIBC). Also, urinary cell-free hsa_circ_0137439 could not only differentiate bladder cancer from normal controls but also distinguish MIBC from non-muscle-invasive bladder cancer (NMIBC). Additionally, hsa_circ_0137439 in urine supernatant could serve as an independent prognostic predicator of recurrence-free survival and overall survival for patients with bladder cancer. Cell assays showed that hsa_circ_0137439 knockdown contributed to the inhibition of cell proliferation and migration via hsa_circ_0137439/miR-142-5p/MTDH axis. In conclusion, urinary cell-free hsa_circ_0137439 could be a promising biomarker for tumor diagnosis and prognostic assessment of bladder cancer patients.
\end{abstract}

Key words: bladder cancer, hsa_circ_0137439, prognosis, biomarker

Bladder cancer is one of the most common urogenital cancers. In 2008, approximately 386,300 people worldwide were diagnosed with bladder cancer, causing nearly 150,200 deaths [1]. Currently, the diagnostic and surveillance strategies for bladder cancer mainly rely on cystoscopy and urine cytology [2]. However, the former is rather invasive, costly, and difficult to detect flat lesions or carcinoma in situ (CIS), whereas the latter is more specific but fails to detect low-grade bladder cancer sensitively. Therefore, identifying novel noninvasive circulating biomarkers with high sensitivity and specificity is extremely important for the diagnosis of bladder cancer [3].

Cell-free circular RNAs (circRNAs) are RNAs formed by back-splicing events as loops, and are found in all types of organisms $[4,5]$. They differ from long noncoding RNA (lncRNA) and microRNAs (miRNAs) as they do not have the 5' and 3' end structures but represent covalently closed cyclic structures [5]. With newly developed technologies of high throughput sequencing and computational approaches, particularly RNA-sequencing, up to 30,000 circRNAs have been identified [6]. CircRNAs are widely involved in the regulation of human physiology and pathology by three main mechanisms including function as a miRNA sponge, a protein-binding molecule and a template for translation into polypeptides $[7,8]$. It is possible that circRNA dysregulation influences the progress of various diseases, including cancer [9]. Importantly, the unique construction of circRNAs makes them insensitive to ribonucleases, which indicates circRNAs can exist in tissues and serum, and as a result could serve as biomarkers for human cancers [10]. Recently, studies have reported that circRNAs could serve as promising biomarkers in numerous types of cancers [11, 12]. Li et al. showed that hsa_circ_0000729 was a potential prognostic biomarker in lung adenocarcinoma [11]. Hsa_ circ_0001445 could regulate the proliferation and migration of hepatocellular cancer and serve as a diagnostic biomarker [12]. However, few studies investigated the diagnostic value of circRNAs in bladder cancer. 
In this study, a novel circRNA hsa_circ_0137439 was firstly screened and analyzed in both normal and bladder cancer urine samples. We found that hsa_circ_0137439 was significantly upregulated in bladder cancer patients and positively correlated with higher tumor stage, higher tumor grade, higher lymph node status and history of MIBC. We further found that hsa_circ_0137439 was also a promising marker for distinguishing bladder cancer patients from normal volunteers and distinguishing NMIBC from MIBC patients. Moreover, we identified that the recurrence-free survival (RFS) and overall survival (OS) of patients with high expression of hsa_circ_0137439 was significantly shorter compared with low expression group. In conclusion, urinary cell-free hsa_circ_0137439 is a promising biomarker for tumor diagnosis and prognostic assessment of bladder cancer patients.

\section{Patients and methods}

Patients. A total of 116 bladder cancer urine samples and 30 normal samples were collected from the First Affiliated Hospital of Anhui Medical University, from December 2016 to May 2018. All samples were verified by histopathology. Tissue samples were stored at $-80^{\circ} \mathrm{C}$ before use. This study was approved by Ethics Committee of First Affiliated Hospital of Anhui Medical University.

Microarray analysis. CircRNA microarray analysis was performed using Human CircRNA Array v2.1 (CapitalBio Technology, China). Total RNA was quantified using NanoDrop ND-1000. The sample preparation and microarray hybridization were performed based on the Arraystar's standard protocols. Briefly, total RNAs were digested with Rnase R (Epicentre Technologies, USA) to remove linear RNAs and enrich circular RNAs. Then, the enriched circRNAs were amplified and transcribed into fluorescent cRNA utilizing a random priming method (Arraystar Super RNA Labeling Kit). The labeled cRNAs were hybridized onto the Arraystar Human circRNA Array V2 (8x15K, Arraystar). After washing the slides, the arrays were scanned by the Agilent Scanner G2505C. Agilent Feature Extraction software (version 11.0.1.1) was used to analyze the acquired array images. Quantile normalization and subsequent data processing was performed using the $\mathrm{R}$ software limma package. Differentially expressed circRNAs were identified through Fold Change filtering. Hierarchical Clustering was performed to show the distinguishable circRNAs expression pattern among samples.

RNA Extraction and qRT-PCR. Total RNA was extracted from bladder cancer urine samples and normal samples using the TRIzol reagent (Invitrogen, Carlsbad, CA, USA) according to the manufacturer's protocol. Total RNA from each specimen was quantified, and quality assurance was conducted using a NanoDrop ND2000 spectrophotometer (NanoDrop, Wilmington, DE, USA). Reaction mixture $(20 \mu \mathrm{l})$ containing $1 \mu \mathrm{g}$ total RNA was reverse transcribed to
cDNA using PrimeScript RT-polymerase (Takara, Dalian, China). qRT-PCR was performed using SYBR-Green Premix Ex Taq (Takara Bio, Nojihigashi, Kusatsu, Japan) and was monitored using the ABI PRISM 7500 Sequence Detection System (Applied Biosystems, Life Technologies, Waltham, MA, USA). The relative expression levels of circRNAs were determined by qRT-PCR. The sequences of the primers used in the qRT-PCR assay are shown. Hsa_circ_0137439 F: 5'-TGTGGATAGGGGAATGA-3'; Hsa_circ_0137439 R: 5'-GGCTGCTTTGCTGTTAC-3'. Gapdh F: 5'-GTCTCCTCTGACTTCAACAGCG-3'; Gapdh R: 5'-ACCACCCTGTTGCTGTAGCCAA-3'. The reaction conditions were as follows: $95^{\circ} \mathrm{C}$ at $5 \mathrm{~min}$ for a preincubation and 40 cycles of $95^{\circ} \mathrm{C}$ for $5 \mathrm{~s}$, annealing temperature of $60^{\circ} \mathrm{C}$ for primer pairs for $30 \mathrm{~s}$, and $72^{\circ} \mathrm{C}$ for $20 \mathrm{~s}$. RNA levels were normalized using $\beta$-actin as the internal control.

Cell culture. The human bladder cancer cell lines, T24 and 5637, were obtained from the ATCC. Cells were cultured in Dulbecco's Modified Eagle Medium (DMED, GIBCO, China) supplemented with 10\% FBS (GIBCO, China) and $1 \%$ penicillin/streptomycin (Life Technologies Inc., USA). All cells were cultured at $37^{\circ} \mathrm{C}$ under $5 \% \mathrm{CO}_{2}$.

Cell viability assay. Cell viability was determined by a 3-(4,5-dimethylthiazol-2-yl)-2, 5-diphenyltetrazolium bromide (MTT, Sigma) assay. In brief, cancer cells were seeded in 96 -well plates in culture medium and incubated in $5 \% \mathrm{CO}_{2}$ at $37^{\circ} \mathrm{C}$. After incubation for the indicated time, $20 \mu \mathrm{l}$ of MTT ( $5 \mathrm{mg} / \mathrm{ml}$ in PBS) was added into each well and incubated for $4 \mathrm{~h}$. The supernatants were carefully aspirated, and $100 \mu$ of dimethyl sulfoxide (DMSO) was added to each well. Absorbance values at $490 \mathrm{~nm}$ were measured on a Microplate Reader (Bio-Rad).

Cell cycle assay. Cells were collected, washed twice with $1 \mathrm{X}$ PBS, and fixed in $70 \%$ ethanol at $-20^{\circ} \mathrm{C}$. After $24 \mathrm{~h}$ of fixation, cells were incubated with RNase A (Takara Bio, Inc., Otsu, Japan) at $100 \mu \mathrm{g} / \mathrm{ml}$ in $1 \mathrm{X}$ PBS for $30 \mathrm{~min}$ at $37^{\circ} \mathrm{C}$. Cells were then stained with propidium iodide (PI; BD Biosciences, San Jose, USA) at $50 \mu \mathrm{g} / \mathrm{ml}$ for $30 \mathrm{~min}$ at room temperature. Subsequently, cells were analyzed for DNA content using a BD FACSCalibur ${ }^{\text {Tit }}$ flow cytometer (BD Biosciences).

Transwell assay. The cell migration ability was analyzed by a Transwell assay, which was performed in 24-well Transwell chambers (Corning, NY, USA). Cells in $200 \mu \mathrm{l}$ serum-free medium were reseeded into the upper chamber. The lower chamber was filled with $600 \mu \mathrm{l}$ medium with $20 \% \mathrm{FBS}$. After incubating for 24 hours, cells on the inner membrane were removed. The outer membrane was fixed with $4 \%$ paraformaldehyde and stained with $0.1 \%$ crystal violet solution. The cells were observed under a microscope and five fields were randomly selected to be photographed.

RNA immunoprecipitation (RIP) assay. RIP experiments were performed using the Magna RIP RNA-Binding Protein Immunoprecipitation Kit (Millipore, Billerica, Massachusetts) according to the manufacturer's instructions. Antibody for RIP assays of AGO2, or control IgG were from Millipore. 
The co-precipitated RNAs were detected by quantitative realtime polymerase chain reaction (qRT-PCR). The total RNAs were the input controls.

Dual-luciferase reporter assay. The dual-luciferase miRNA Target Expression vector pmirGLO (Promega, Madison, Wisconsin) was used to generate luciferase reporter constructs. Full length hsa_circ_0137439 sequence with wildtype (WT) and mutant type (Mut) miRNA binding site were obtained from Vigene Biosciences (Rockville, Maryland). Cells were seeded in 96-well plates and co-transfected with wild-type or mutated circRNA constructs and miR-142-5p mimic. Luciferase activity was measured with the dual-luciferase reporter assay system (Promega). Firefly luciferase activity was normalized against Renilla luciferase activity.

Statistical analysis. Statistical differences between independent groups were calculated by Mann-Whitney $U$ test, Kruskal-Wallis test or Student's t-test, as appropriate. AUC values, sensitivity, and specificity for cell-free hsa_ circ_0137439 were quantified by using receiver operating characteristic (ROC) analysis to assess its diagnostic efficiency in differentiating patients with bladder cancer from healthy controls. The optimal cutoff thresholds for diagnosis were obtained by Youden index [14]. Patients were equally divided into two groups based on their relative expression. RFS and OS curves were evaluated by the Kaplan-Meier method and the survival differences of patients in sub-groups were estimated by the log-rank test. Median expression values were selected as cutoffs among groups analyzed. All graphing and statistical analyses were carried out using SPSS 17.0. A p-value $<0.05$ (two-tailed test) was regarded as significant difference.

\section{Results}

Hsa_circ_0137439 was upregulated in bladder cancer samples. To discover the potential circRNAs associated with bladder cancer, urine samples from 10 healthy controls and 10 bladder cancer patients were sent for microarray. As shown in Figure 1A, a total of 53 circRNAs were upregulated and 33 circRNAs were downregulated in bladder cancer patients. Of the most significantly changed circRNAs, hsa_circ_0137439 was filtered and the expression level is shown as Figure 1. The location of hsa_circ_0137439 is chr8:98673299-98708521, and the host gene is MTDH. The genome length is 35222 bp and the spliced length is 5772 bp. To further evaluate the potential significance of hsa_circ_0137439, a total of 30 normal controls and 116 bladder cancer samples were examined. As shown in Figure 1C, hsa_circ_0137439 was also remarkably increased in bladder cancer patients, which indicates that hsa_circ_0137439 is a potential bladder cancer associated circRNA.
A

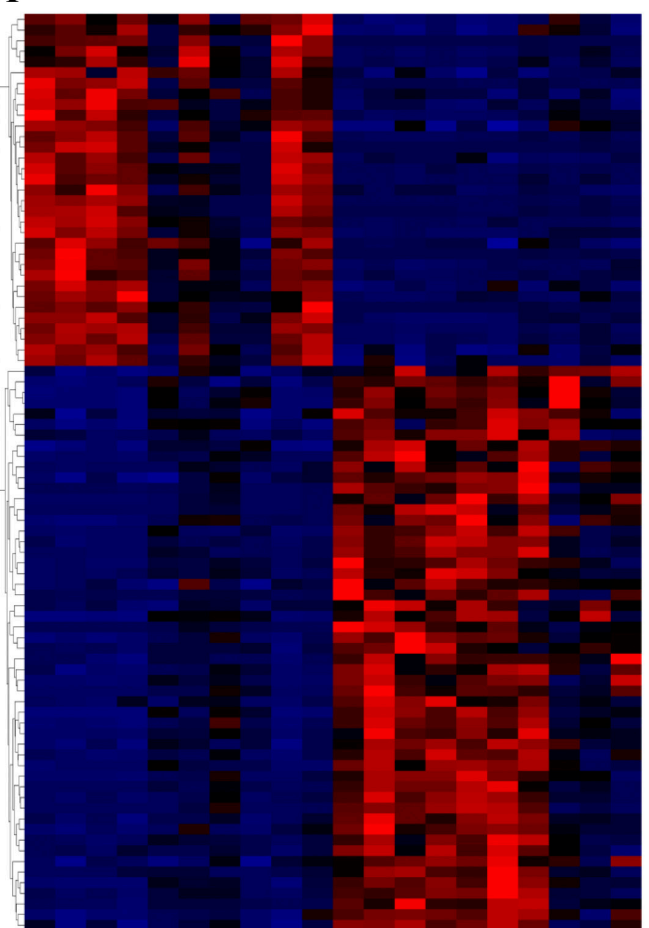

B
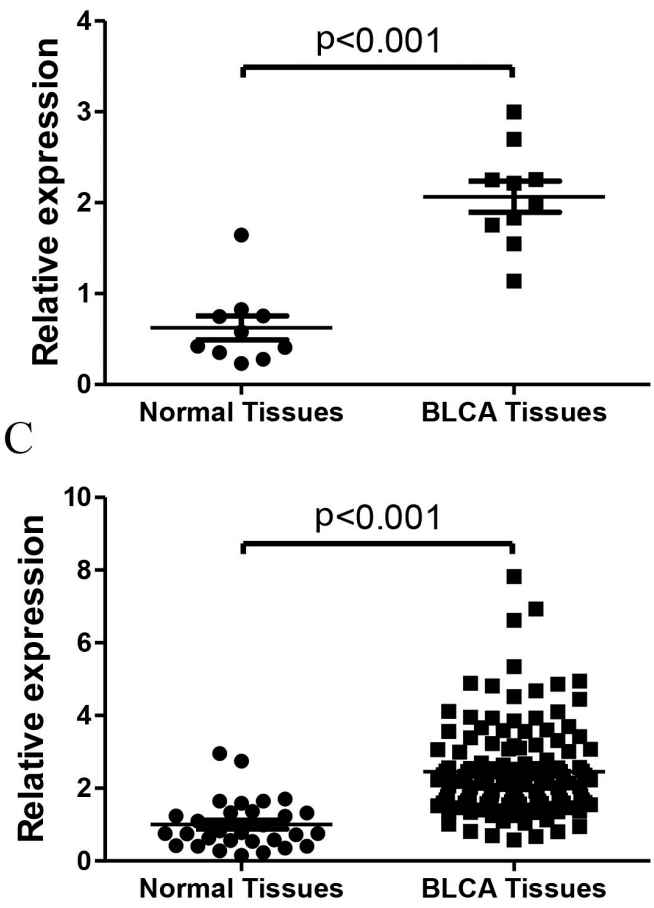

Figure 1. Hsa_circ_0137439 was upregulated in bladder cancer urine samples. A) The cluster heat map shows the upregulated and downregulated circRNAs in bladder cancer samples. Red color indicates high expression level and blue color indicates low expression level. B) The relative expression level of hsa_circ_0137439 in the 10 normal control and 10 bladder cancer samples. C) The relative expression level of hsa_circ_0137439 in the total 30 normal control and 116 bladder cancer samples. 
Baseline characteristics and hsa_circ_0137439 expression of the 116 bladder cancer patients. The baseline characteristics of the 116 bladder cancer patients are shown in Table 1 . The average age was 59.08 years, and $76.72 \%$ of the patients were male. To further investigate whether high expression of hsa_circ_0137439 was related to the clinical progression of bladder cancer, we analyzed the expression of hsa_circ_0137439 with the clinicopathological features. The expression of hsa_circ_0137439 showed no significant difference when age, gender and history of smoking were analyzed. However, increased expression of hsa_circ_0137439 was found in higher tumor stage $(\mathrm{p}<0.001)$, higher grade $(p<0.001)$, higher lymph node status $(p=0.035)$ and MIBC patients $(\mathrm{p}<0.001)$. These data suggested that upregulation of hsa_circ_0137439 was associated with malignant biological behaviors of bladder cancer patients.

Diagnostic efficacy of urinary cell-free hsa circ_0137439. Since the expression of hsa_circ_0137439 was increased in bladder cancer samples and correlated with malignant biological behaviors, we further examined the diagnostic efficacy of urinary cell-free hsa_circ_0137439. As shown in Figure 2A, ROC curve analyses presented that urinary cell-free hsa_circ_0137439 could serve as a forceful

Table 1. Urinary cell-free hsa_circ_0137439 expression and clinicopathologic parameters in bladder cancer patients.

\begin{tabular}{lcccc}
\hline Variables & $\begin{array}{c}\text { Number } \\
\text { of cases }\end{array}$ & Percentage & $\begin{array}{c}\text { Relative expression } \\
\text { Mena (95\% CI) }\end{array}$ & p-value \\
\hline Age & & & & 0.938 \\
$\quad \leq 60$ & 63 & 54.31 & $2.417(2.148-2.686)$ & \\
$>60$ & 53 & 45.69 & $2.435(2.039-2.831)$ & \\
Gender & & & & 0.347 \\
Male & 89 & 76.72 & $2.485(2.212-2.759)$ & \\
Female & 27 & 23.26 & $2.227(1.812-2.642)$ & \\
Tumor stage & & & & $<0.001$ \\
Ta & 21 & 18.10 & $1.611(1.252-1.969)$ & \\
T1 & 20 & 17.24 & $1.811(1.540-2.081)$ & \\
T2 & 23 & 19.83 & $2.193(1.786-2.600)$ & \\
T3 & 29 & 25 & $3.059(2.589-3.531)$ & \\
T4 & 23 & 19.83 & $3.291(2.626-3.956)$ & \\
Tumor Grade & & & & $<\mathbf{0 . 0 0 1}$ \\
LOW & 53 & 45.69 & $1.978(1.673-2.283)$ & \\
HIGH & 63 & 54.31 & $2.802(2.491-3.113)$ & \\
Lymph node status & & & & $\mathbf{0 . 0 3 5}$ \\
N0 & 71 & 61.21 & $2.289(2.094-2.484)$ & \\
N1 & 18 & 15.52 & $3.034(2.484-3.921)$ & \\
N2 & 13 & 11.21 & $2.568(1.732-3.404)$ & \\
N3 & 9 & 7.76 & $3.365(2.310-4.420)$ & \\
N4 & 5 & 4.31 & $2.844(0.781-4.906)$ & \\
N/MIBC & & & & $<\mathbf{0 . 0 0 1}$ \\
MIBC & 54 & 46.55 & $2.954(2.620-3.288)$ & \\
NMIBC & 62 & 53.45 & $1.996(1.718-2.274)$ & \\
\hline
\end{tabular}

MIBC (muscle-invasive bladder cancer); NMIBC (non-muscle invasive bladder cancer) noninvasive biomarker for distinguishing bladder cancer patients from healthy controls with an AUC value of 0.890 (95\% CI $=0.818-0.962)$. At the cut-off value of 1.360 , the sensitivity was $87.93 \%$ and the specificity was $80.06 \%$. We also evaluated the diagnostic value of differentiating NMIBC from MIBC. Data showed that the cell-free hsa_circ_0137439 expression in the urine manifested an AUC value of 0.798 with a sensitivity of $88.56 \%$ and a specificity of $73.45 \%$ at a cut-off value of 0.628 for identifying NMIBC from MIBC (Figure 2B).

Association of urinary cell-free hsa_circ_0137439 expressions with the prognosis of bladder cancer patients. To further evaluate the clinical significance of cell-free hsa circ_0137439, the association between hsa_circ_0137439 and RFS (recurrence-free survival) and OS (overall survival) was analyzed. As shown in Figure 3, Kaplan-Meier survival curves manifested that bladder cancer patients with high urinary cell-free hsa_circ_0137439 expressions had significantly worse RFS (Figure 3A) and OS (Figure 3B) than those with low expression, indicating that cell-free hsa circ_0137439 was a potential biomarker for predicting the prognosis of bladder cancer.

Knockdown of hsa_circ_0137439 suppressed proliferation and metastasis of bladder cancer cells. To further investigate the mechanism of hsa_circ_0137439 in bladder cancer, we silenced hsa_circ_0137439, and the effect on the proliferation and metastasis of bladder cancer cell lines was detected (Figure 4A). The proliferation ability of T24 and 5637 cell lines was examined by MTT assay (Figure 4B) and cell cycle changes by flow cytometric analysis of PI histograms (Figure 4C). We found that knockdown of hsa_circ_0137439 significantly inhibited the proliferation of both cell lines via induction of G1 phase cell cycle arrest. Transwell assay also presented that silencing hsa_circ_0137439 suppressed cell migration of T24 and 5637 cell lines (Figure 4D). In conclusion, our results suggested that knockdown of hsa_circ_0137439 suppressed proliferation and metastasis of bladder cancer cells.

Hsa_circ_0137439 could function as ceRNA via directly sponging miR-142-5p. To explore the mechanism of hsa_circ_0137439 on the progression and metastasis of bladder cancer, we firstly explored the cellular location of hsa_circ_0137439. As shown in Figure 5A, we found that hsa_circ_0137439 was mainly located in cytoplasm, which indicated that hsa_circ_0137439 might function as ceRNA via directly sponging miRNA. A total of 5 circRNAs were predicted based on interactome [13], and the correlations between circRNA and miRNAs were evaluated. As shown in Figure 5B, only miR-142 was negatively correlated with hsa_circ_0137439 expression, which indicted that hsa_circ_0137439 might function as ceRNA via directly sponging miR-142-5p. Because Ago2 is a key component of RNA induced silencing complexes [14], immunoprecipitation was performed and both hsa_circ_0137439 and miR-142 could be pulled down by Ago2 antibody (Figure 5C). The 
$\mathbf{A}$

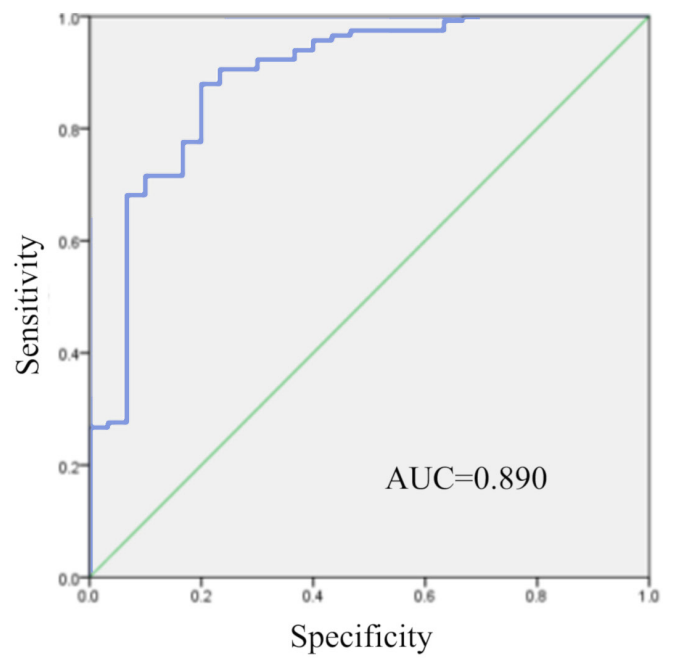

B

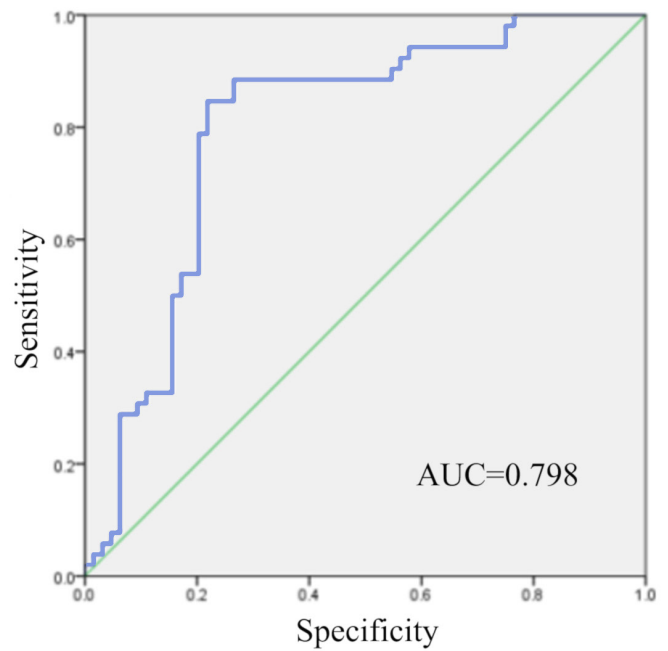

Figure 2. ROC curve analysis based upon urinary cell-free hsa circ 0137439 to evaluate the diagnostic value. A) Urinary cell-free hsa circ 0137439 yielded an AUC value of 0.890 with $\mathbf{8 7 . 9 3 \%}$ sensitivity and $\mathbf{8 0 . 0 6 \%}$ specificity in discriminating bladder cancer patients $(\mathrm{n}=116)$ from healthy control subjects $(n=30)$. B) Urinary cell-free hsa_circ_0137439 generated an AUC value of 0.798 with $88.56 \%$ sensitivity and $73.45 \%$ specificity in distinguishing NMIBC patients $(n=62)$ from MIBC patients $(n=54)$.

A

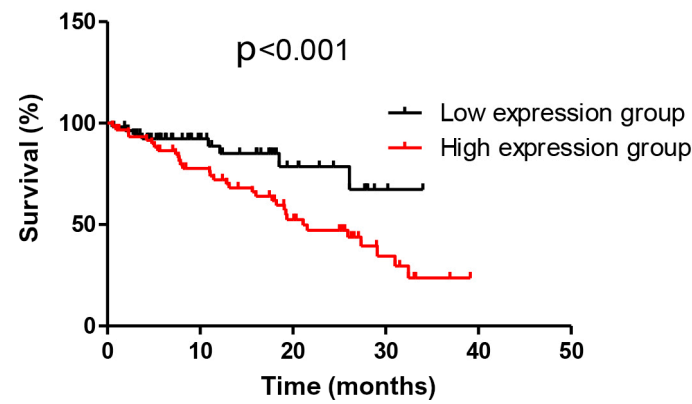

B

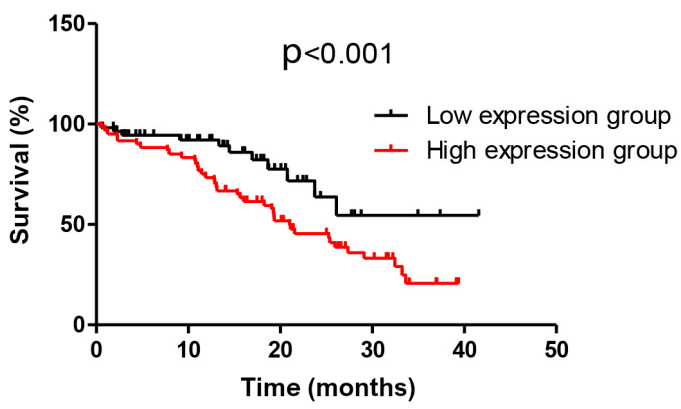

Figure 3. Kaplan-Meier survival analysis of bladder cancer patients according to urinary cell-free hsa_circ_0137439 expression. A) The RFS rate of bladder cancer patients according to cell-free hsa_circ_0137439 expression. B) The OS rate of bladder cancer patients according to cell-free hsa_ circ_0137439 expression. The log-rank test was used to compare the differences between subgroups.

miR-142-5p binding sequence on circRNA is shown as Figure 5D (upper panel), and the wild type (Wt) and mutant type (Mut) of binding sequence are shown in lower panel. Then dual reporter luciferase assay was performed in $293 \mathrm{~T}$ cells co-transfected with both circRNA and miR-142 mimics based on gradient method. As shown in Figure 5E, with the increasing amount of transfected miR-142 mimics, luciferase activities of circRNA-wt were significantly decreased. However, luciferase activities of circRNA-mut had no obvious change. Moreover, after circRNA knockdown and miR-142-5p overexpression, we found the expression of hsa_circ_0137439 and miR-142-5p showed negative correlation (Figure 5F). In conclusion, our results suggested that hsa_circ_0137439 could function as ceRNA via directly sponging miR-142-5p.
Hsa_circ_0137439 promoted the proliferation and metastasis of bladder cancer cells via hsa_circ_0137439/ miR-142-5p/MTDH axis. Since our data showed that miR-142-5p was a direct target of hsa_circ_0137439, we then explored the functions and mechanisms of miR-142-5p on the proliferation and metastasis of bladder cancer. As shown in Figure 6A, the inhibition of miR-142-5p increased hsa_circ_0137439 level in both cell lines. To evaluate the function of miR-215, cells were transfected with hsa_circ_0137439 siRNA and/or miR-142-5p, then MTT and Transwell assay were performed. As shown in Figure $6 \mathrm{~B}$ and $6 \mathrm{C}$, miR-142-5p could repress the functions of hsa_circ_0137439 in both proliferation and metastasis, which indicated that miR-142-5p was a functional target of hsa_circ_0137439. Furthermore, bioinformatics predic- 
A

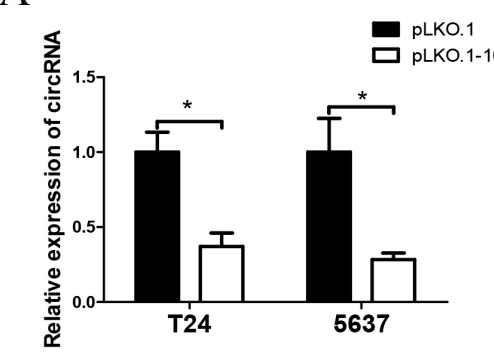

$\mathrm{B}$
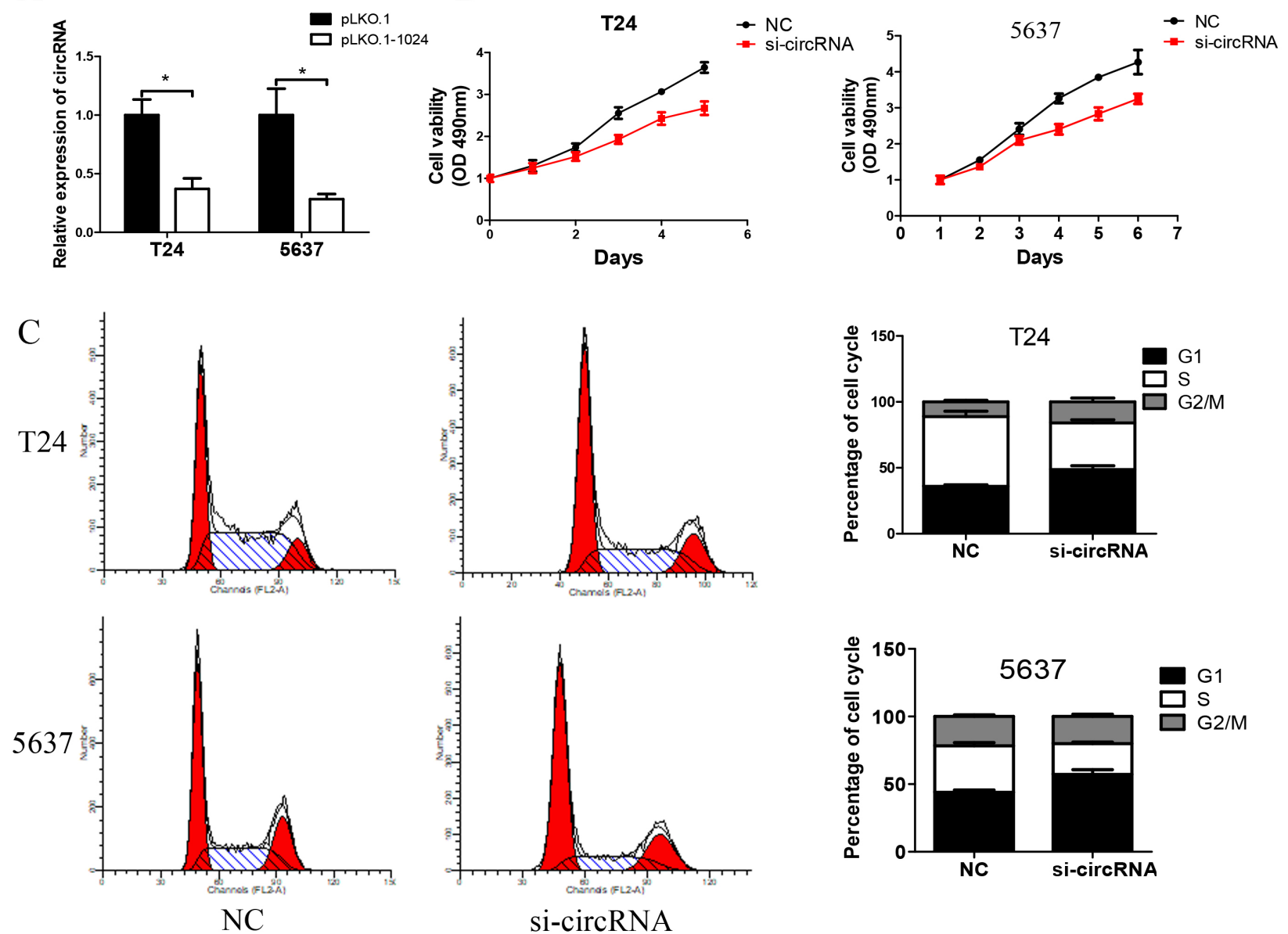

$\mathrm{D}$

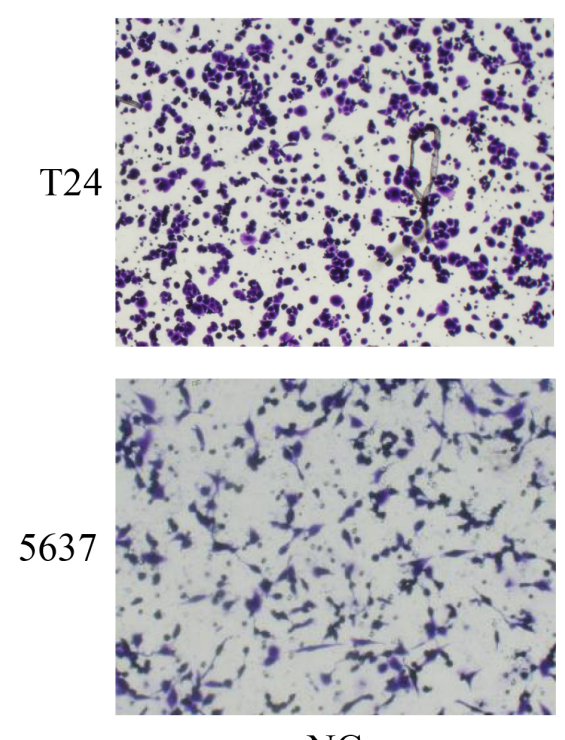

$\mathrm{NC}$
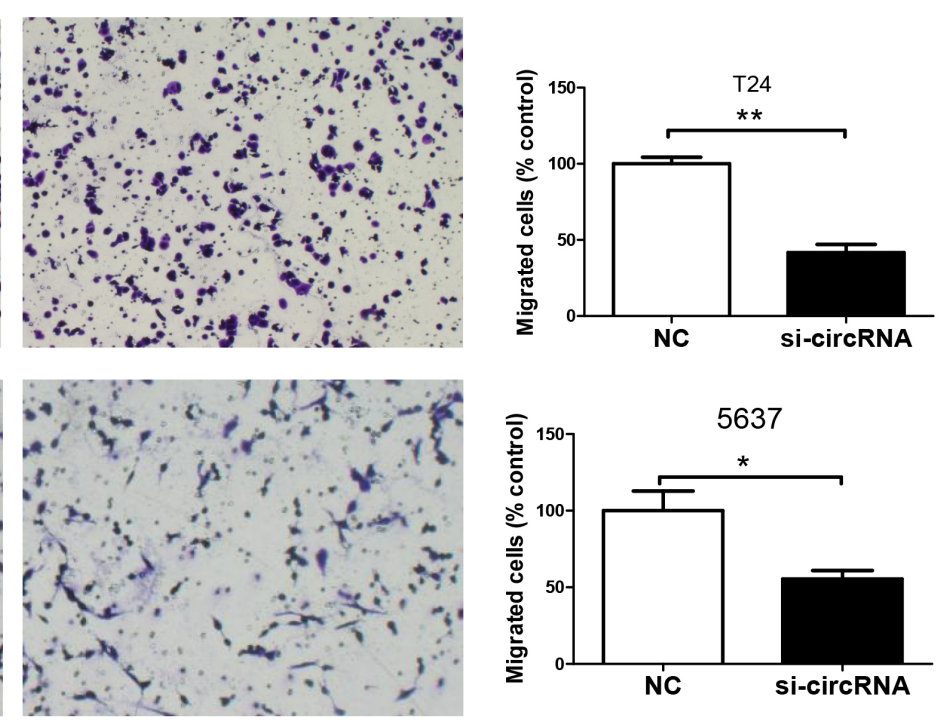

Figure 4. Effect of hsa_circ_0137439 knockdown on the proliferation and migration of bladder cancer cells. A) The efficiency of hsa_circ_0137439 knockdown. B) Effect of hsa_circ_0137439 knockdown on the proliferation on bladder cancer cells based on MTT assay. C) Flow cytometry verified the cell cycle of T24 and 5637 cells. D) Transwell assay was performed to evaluate effect of hsa_circ_0137439 knockdown on cell migration. ${ }^{*}$ p $<0.05$, ${ }^{* *} \mathrm{p}<0.01,{ }^{* * *} \mathrm{p}<0.001$. 
A

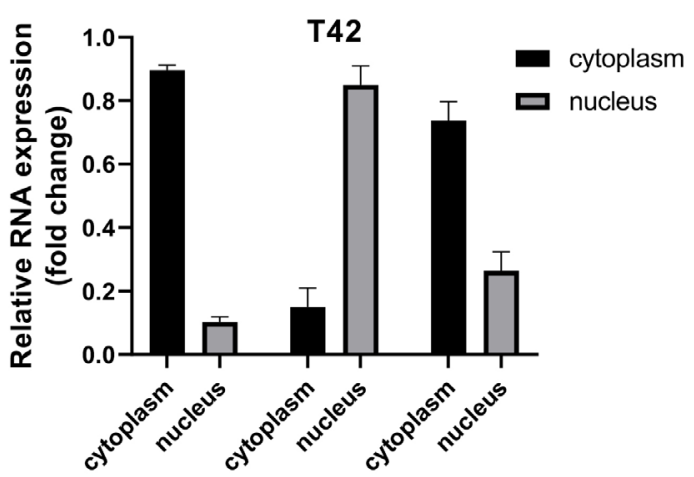

B

D

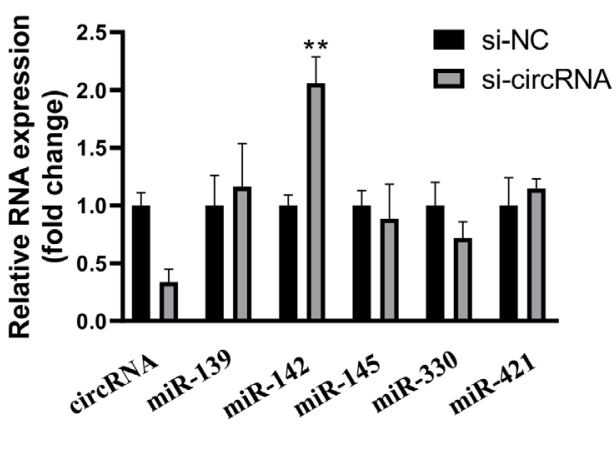

UAGAAAAAUUUUAACUUUAUUU hsa_circ_0137439 | | | | | |

CAUCACGAAAGAUGAAAUAC miR-142-5p

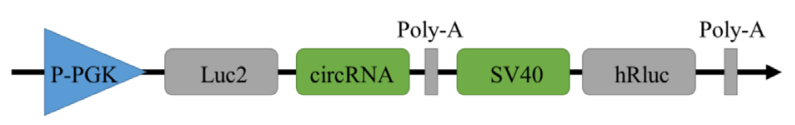

UAGAAAAAUUUAACUUUUAUU circRNA WT

\section{| | | | |}

UAGAAAAAUUUATGAAATAU circRNA Mut

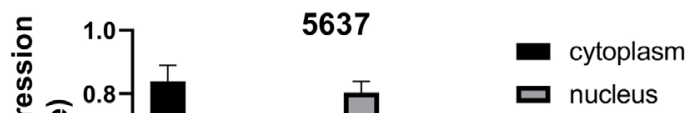

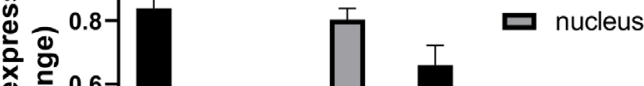

$\frac{1}{\mathrm{D}}$

1)

$\sum_{\substack{\pi \\ 0}}^{0} 0.2$

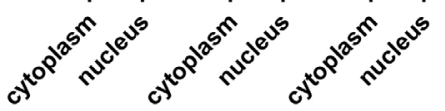

C 。 250 293T

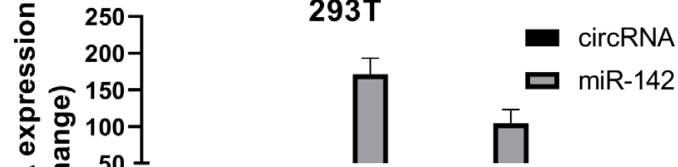

¿돈 25

$\gg$ 車

要

$0-1$

IgG Input Ago2

$\mathbf{E}$

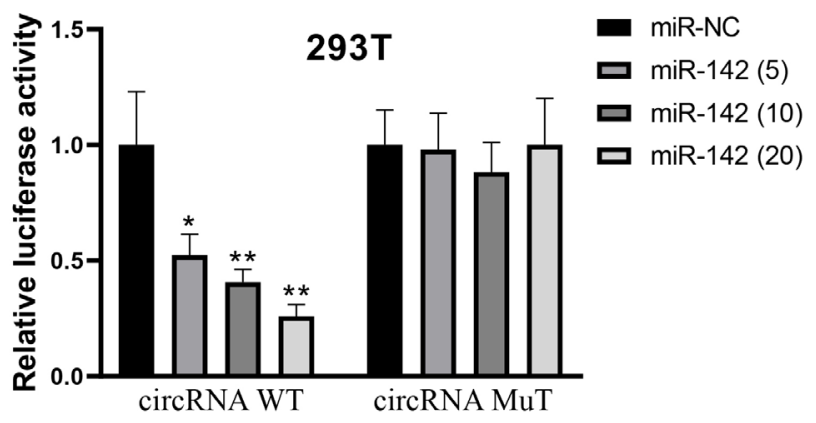

$\mathbf{F}$
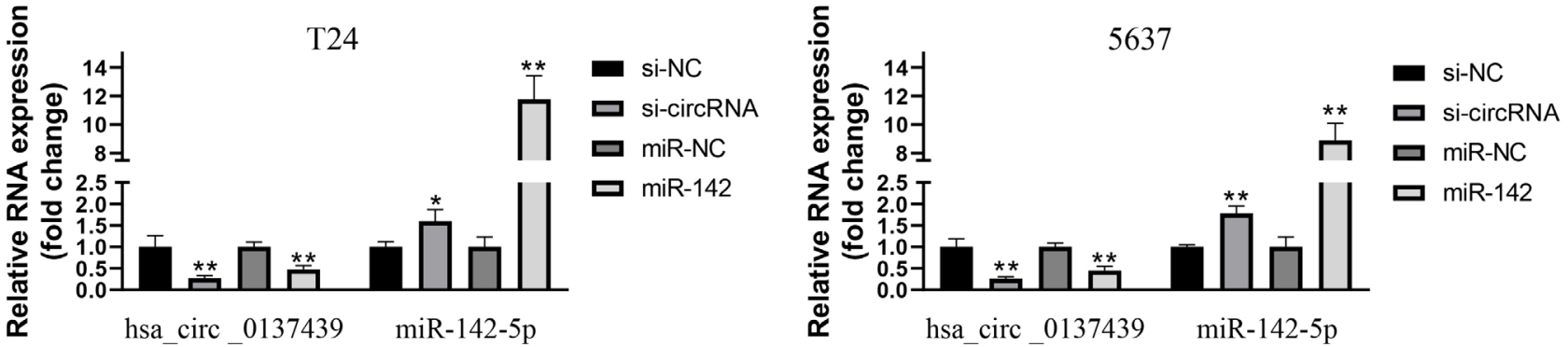

Figure 5. Hsa circ 0137439 functioned as a ceRNA via directly sponging miR-142-5p. A) Detection of subcellular location of hsa circ 0137439 in both T24 and 5637 cell lines. B) The expression correlations between hsa_circ_0137439 and potential target miRNAs. C) Anti-Ago2 RIP assay verified the combination between miR-142-5p and hsa_circ_0137439. D) The predicted targeting sequence of miR-142-5p on the hsa_circ_0137439 (upper panel); wild-type (Wt) and mutant type (Mut) of binding sites. E) The luciferase reporter assay verified that hsa_circ_0137439 functioned as a ceRNA via direct sponging miR-142-5p. F) Effect of circRNA knockdown and miRNA overexpression on expression of both hsa_circ_0137439 and miR-142-5p. ${ }^{\star} \mathrm{p}<0.05$, ${ }^{* *} \mathrm{p}<0.01,{ }^{* * *} \mathrm{p}<0.001$. 
A

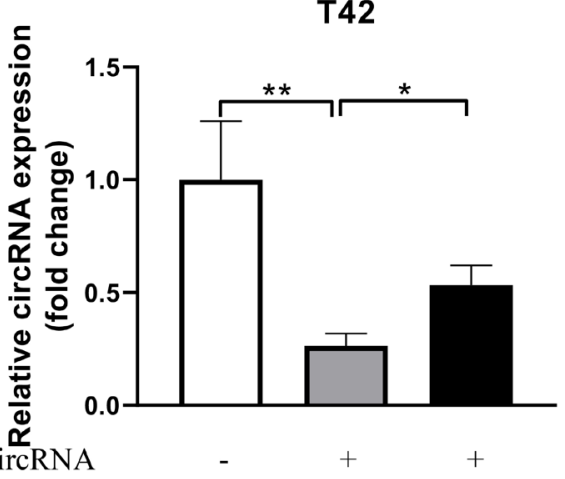

5637

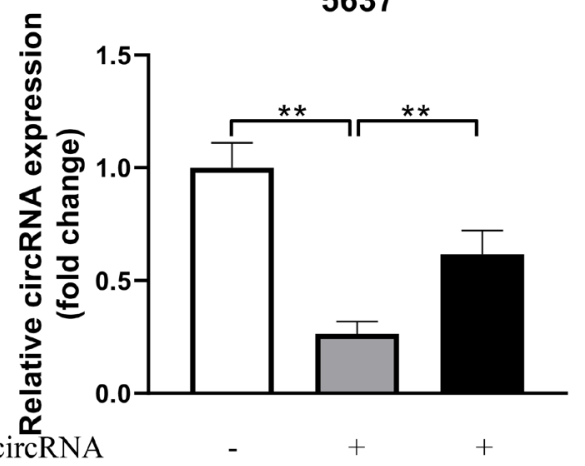

si-circRNA

miR-142 inhibitor

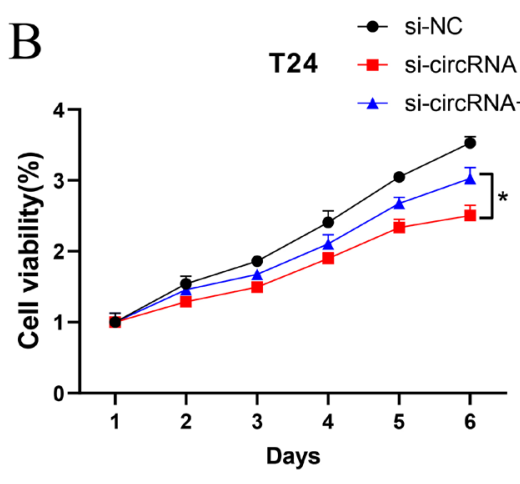

$\mathrm{C}$

T42

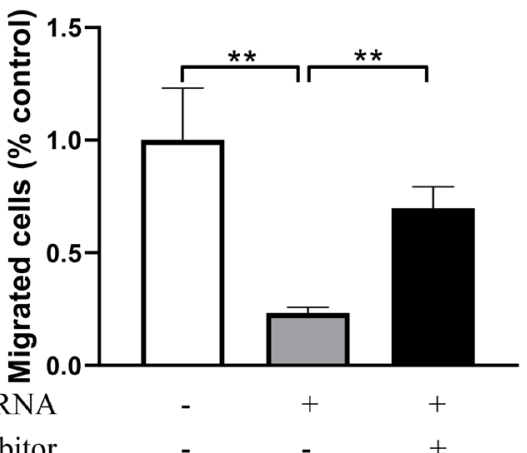

miR-142 inhibitor

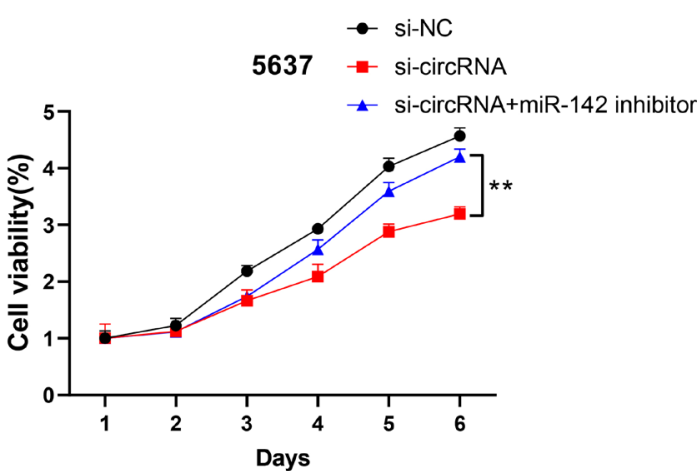

5637

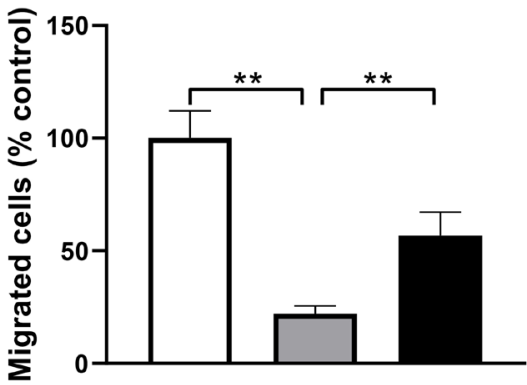

si-circRNA miR-142 inhibitor

miR-142 inhibitor
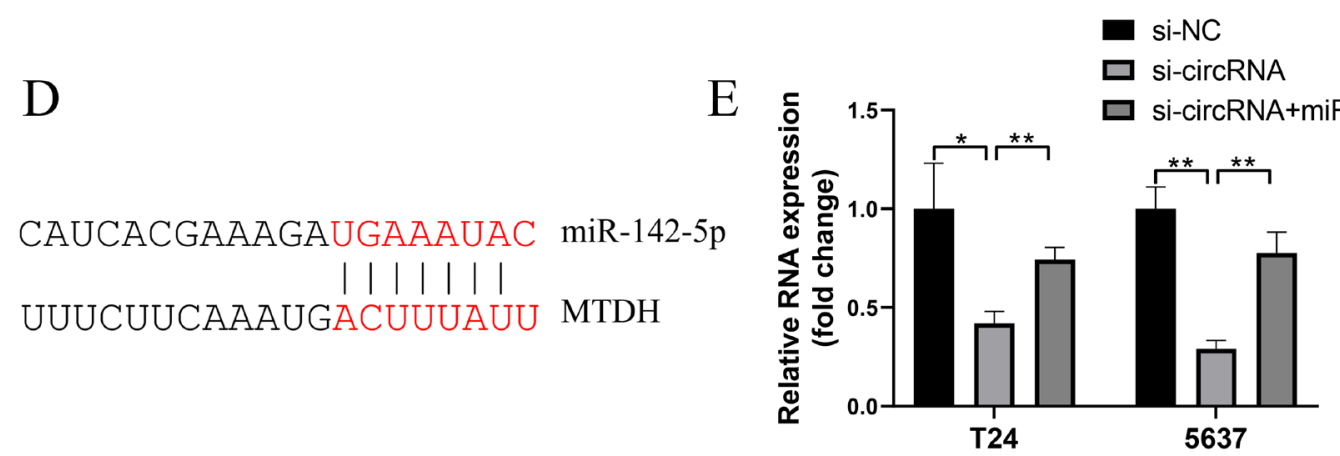

Figure 6. Hsa circ 0137439 promoted the proliferation and metastasis of breast cancer cells via hsa circ 0137439/miR-142-5p/MTDH axis. A) Expression of hsa_circ_0137439 after hsa_circ_0137439 and miR-215 inhibition. B) Effect of hsa_circ_0137439 and miR-142-5p inhibition on cell proliferation. C) Effect of hsa_circ_0137439 and miR-142-5p inhibition on cell migration. D) The predicted correlation between miR-142-5p and hsa circ_0137439. E) Expression of MTDH after hsa_circ_0137439 and miR-142-5p inhibition. ${ }^{*} p<0.05,{ }^{* *} p<0.01,{ }^{* * *} p<0.001$. 
tion showed the potential combination of miR-142-5p with MTDH (Figure 6D). Then mRNA expression level of MTDH was detected with real-time PCR. As shown in Figure 6E, knockdown of hsa_circ_0137439 could significantly decrease the expression of MTDH, and miR-142-5p inhibitor increased MTDH expression level. Taken together, our results suggested that hsa_circ_0137439 could function via hsa_circ_0137439/miR-142-5p /MTDH2 axis.

\section{Discussion}

The identification of molecular markers in body fluids (e.g., serum and urine), which can be used as non-invasive diagnostic, prognostic and surveillance markers in cancer management, is one of the most ambitious challenges in oncology research [15]. To date, the increased interest in non-invasive biomarkers, allowed by the use of novel methodologies (such as next generation sequencing, single-cell sequencing approaches, and digital PCR) has greatly improved the translational potential of researches into clinical application $[16,17]$. Recently, circRNAs have been considered as potential biomarkers in many kinds of tumors due to its stability in body fluids [10], such as in lung cancer $[11,18]$, pancreatic cancer [19], gastric cancer [20], and so on.

Based on previously studies, we thought that circRNAs might serve as novel biomarkers in cancers. However, most of studies mainly focused on the potential functions, and their clinical diagnostic value remains largely unknown, specifically in bladder cancer. In our study, we firstly found that hsa_circ_0137439 was remarkably upregulated in 10 bladder cancer samples compared with 10 normal controls based on microarray, which was further verified in 116 bladder cancer and 30 normal samples $(p<0.001)$ by quantitative RT-PCR assays. Clinicopathological features showed that the overexpression of hsa_circ_0137439 level was negatively associated with higher tumor stage, higher tumor grade, higher lymph node status, and history of MIBC, indicating hsa_circ_0137439 might contribute to malignant behaviors of bladder cancer. We also evaluated whether hsa_circ_0137439 could act as a valuable biomarker for the diagnosis of bladder cancer. As shown in Figure 2, we demonstrated that hsa_circ_0137439 could distinguish not only bladder cancer patients from normal controls but also distinguish NMIBC from MIBC patients. Based on KaplanMeier method, we also found that hsa_circ_0137439 could predict the prognosis of both RFS and OS in bladder cancer patients, in which high expression of hsa_circ_0137439 suggested poor prognosis.

Also in this study, we took further steps to ectopically silence hsa_circ_0137439 in bladder cancer cells and then evaluated the functional role of hsa_circ_0137439 inhibition in cancer malignant behaviors. Then, through several in vitro assays, we showed that hsa_circ_0137439 inhibition significantly suppressed both proliferation via induction of G1 phase cell cycle arrest and migration abilities (Figure 4). Based on our results, we also suggested that hsa_circ_0137439 could influence the malignant behaviors of bladder cancer cells.

Previous studies have reported that the possible mechanisms of circRNAs are closely associated with the location of circRNAs in cells [21]. For example, lncRNAs that were located in cytoplasm might function as a miRNA sponge that interdicted the effects of miRNAs [22]. Based on the results of qRT-PCR, we found that hsa_circ_0137439 was mainly located in cytoplasm, which indicated that the circRNA might also function as a ceRNA. Bioinformatics analysis showed a potential binding site to miR-142-5p. We found hsa_circ_0137439 knockdown increased miR-142-5p expression in bladder cancer cells. Further dual-luciferase reporter assays and RIP assay confirmed the binding of miR-142-5p to hsa_circ_0137439. The miR-142-5p has been reported as a functional miRNA that could influence proliferation and migration in cancers, including pancreatic cancer, non-small cell lung cancer, osteosarcoma, and so on [23-25]. These previous studies reported that inhibition of miR-142-5p by hsa_circ_0137439 could suppress the proliferation and migration of breast cancer cells, which was coincided with our function experiments. Moreover, we found that MTDH, a crucial proto-oncogene in tumorigenesis, was be targeted by miR-142-5p.

In conclusion, our study found a novel biomarker, which was positively correlated with malignant clinical characteristic of patients, could distinguish bladder cancer from normal control and predict the prognosis of both RFS and OS. Moreover, we found that hsa_circ_0137439/ miR-142-5p/MTDH axis was a tumor regulator, which contributed to the proliferation and migration of bladder cancer cells. In the future, we believe that by further studying the functions of circRNAs, we could improve our understanding of the mechanisms of disease associated with circRNAs and improve the diagnosis and prevention of circRNAs-associated diseases.

\section{References}

[1] JEMAL A, BRAY F, CENTER MM, FERLAY J, WARD E et al. Global cancer statistics. CA Cancer J Clin 2011; 61: 69-90. https://doi.org/10.3322/caac.20107

[2] VROOMAN OP, WITJES JA. Urinary markers in bladder cancer. Eur Urol 2008; 53: 909-916. https://doi.org/10.1016/j. eururo.2007.12.006

[3] KAMAT AM, VLAHOU A, TAYLOR JA, HUDSON ML, PESCH B et al. Considerations on the use of urine markers in the management of patients with high-grade non-muscleinvasive bladder cancer. Urol Oncol 2014; 32: 1069-1077. https://doi.org/10.1016/j.urolonc.2014.06.017

[4] Ji W, Qiu C, Wang M, Mao N, Wu S, Dai Y. Hsa_circ_0001649: A circular RNA and potential novel biomarker for colorectal cancer Biochem Biophys Res Commun 2018; 497: 122-126. https://doi.org/10.1016/j.bbrc.2018.02.036 
[5] YANG D, SUN L, LI Z, GAO P. Noncoding RNAs in Regulation of Cancer Metabolic Reprogramming. Adv Exp Med Biol 2016; 927: 191-215. https://doi.org/10.1007/978-98110-1498-7_7

[6] HAN YN, XIA SQ, ZHANG YY, ZHENG JH, LI W. Circular RNAs: A novel type of biomarker and genetic tools in cancer. Oncotarget 2017; 8: 64551-64563. https://doi.org/10.18632/ oncotarget. 18350

[7] LI Z, HUANG C, BAO C, CHEN L, LIN M et al. Exon-intron circular RNAs regulate transcription in the nucleus. Nat Struct Mol Biol 2015; 22: 256-264. oi: 10.1038/nsmb.2959

[8] GRANADOS-RIVERON JT, AQUINO-JARQUIN G. The complexity of the translation ability of circRNAs. Biochim Biophys Acta 2016; 1859: 1245-1251. https://doi. org/10.1016/j.bbagrm.2016.07.009

[9] SZABO L, SALZMAN J. Detecting circular RNAs: bioinformatic and experimental challenges. Nat Rev Genet 2016; 17: 679-692. https://doi.org/10.1038/nrg.2016.114

[10] Chen Y, Li C, Tan C, Liu X. Circular RNAs: a new frontier in the study of human diseases. J Med Genet 2016; 53: 359-365. https://doi.org/10.1136/jmedgenet-2016-103758

[11] LI S, SUN X, MIAO S, LU T, WANG Y et al. hsa_ circ_0000729, a potential prognostic biomarker in lung adenocarcinoma. Thorac Cancer 2018; 9: 924-930. https://doi. org/10.1111/1759-7714.12761

[12] ZHANG X, ZHOU H, JING W, LUO P, QIU S et al. The Circular RNA hsa_circ_0001445 Regulates the Proliferation and Migration of Hepatocellular Carcinoma and May Serve as a Diagnostic Biomarker. Dis Markers 2018; 2018: 3073467. https://doi.org/10.1155/2018/3073467

[13] DUDEKULA DB, PANDA AC, GRAMMATIKAKIS I, DE S, ABDELMOHSEN K et al. CircInteractome: A web tool for exploring circular RNAs and their interacting proteins and microRNAs. RNA Biol 2016; 13: 34-42. https://doi.org/10.10 80/15476286.2015.1128065

[14] KONG X, DUAN Y, SANG Y, LI Y, ZHANG H et al. LncRNA-CDC6 promotes breast cancer progression and function as ceRNA to target CDC6 by sponging microRNA- 215 . J Cell Physiol 2019; 234: 9105-9117. https://doi.org/10.1002/ jcp. 27587

[15] PASTORE AL, PALLESCHI G, SILVESTRI L, MOSCHESE D, RICCI S ET AL. Serum and urine biomarkers for human renal cell carcinoma. Disease markers 2015; 2015: 251403. https://doi.org/10.1155/2015/251403
[16] LUO X, YANG B, NATTEL S. MicroRNAs and atrial fibrillation: mechanisms and translational potential. Nat Rev Cardiol 2015; 12: 80-90. https://doi.org/10.1038/nrcardio.2014.178

[17] FENDLER A, STEPHAN C, YOUSEF GM, KRISTIANSEN G, JUNG K. The translational potential of microRNAs as biofluid markers of urological tumours. Nat Rev Urol 2016; 13: 734-752. https://doi.org/10.1038/nrurol.2016.193

[18] HANG D, ZHOU J, QIN N, ZHOU W, MA H et al. A novel plasma circular RNA circFARSA is a potential biomarker for non-small cell lung cancer. Cancer Med 2018; 7: 2783-2791. https://doi.org/10.1002/cam4.1514

[19] YANG F, LIU DY, GUO JT, GE N, ZHU P et al. Circular RNA circ-LDLRAD3 as a biomarker in diagnosis of pancreatic cancer. World J Gastroenterol 2017; 23: 8345-8354. https://doi.org/10.3748/wjg.v23.i47.8345

[20] SUN H, TANG W, RONG D, JIN H, FU K et al. Hsa circ_0000520, a potential new circular RNA biomarker, is involved in gastric carcinoma. Cancer Biomark. 2018; 21: 299-306. https://doi.org/10.3233/CBM-170379

[21] QUAN M, CHEN J, ZHANG D. Exploring the secrets of long noncoding RNAs. Int J Mol Sci 2015; 16: 5467-5496. https://doi.org/10.3390/ijms16035467

[22] SANG Y, CHEN B, SONG X, LI Y, LIANG Y et al. circRNA_0025202 Regulates Tamoxifen Sensitivity and Tumor Progression via Regulating the miR-182-5p/FOXO3a Axis in Breast Cancer. Mol Ther 2019; 27: 1638-1652. https://doi. org/10.1016/j.ymthe.2019.05.011

[23] WANG Z, LIU Z, FANG X, YANG H. MiR-142-5p Suppresses Tumorigenesis by Targeting PIK3CA in Non-Small Cell Lung Cancer. Cell Physiol Biochem 2017; 43: 25052515. https://doi.org/10.1159/000484459

[24] YAO R, XU L, WEI B, QIAN Z, WANG J et al. miR-142-5p regulates pancreatic cancer cell proliferation and apoptosis by regulation of RAP1A. Pathol Res Pract 2019; 215: 152416. https://doi.org/10.1016/j.prp.2019.04.008

[25] CHENG D, LI J, ZHANG L, HU L. miR-142-5p suppresses proliferation and promotes apoptosis of human osteosarcoma cell line, HOS, by targeting PLA2G16 through the ERK1/2 signaling pathway. Oncol Lett 2019; 17: 1363-1371. https://doi.org/10.3892/ol.2018.9712 